\title{
Spatial-temporal patterns of urban anthropogenic heat discharge in Fuzhou, China observed from sensible heat flux using Landsat TM/ETM+ data
}

\author{
Youshui Zhang $^{\mathrm{a}, *}$, Heiko Balzter ${ }^{\mathrm{b}}$, Xiongchang $\mathrm{Wu}^{\mathrm{a}}$ \\ ${ }^{a}$ College of Geography, Fujian Normal University, Fuzhou 350007, P.R.China \\ ${ }^{\mathrm{b}}$ Centre for Landscape and Climate Research \& Department of Geography, University of \\ Leicester, University Road, Leicester, LE1 7RH, UK
}

\begin{abstract}
The urban heat island effect describes the phenomenon of increased surface temperatures in urban environments compared to their surroundings. It is linked to decreased vegetation cover, high proportions of artificial impervious surfaces and anthropogenic heat discharge. This paper evaluates the surface heat balance to clarify the contribution of anthropogenic heat discharges to the urban thermal environment. We use a heat-balance model and satellite images (Landsat TM and ETM+ images acquired in 1989 and 2001), together with meteorological station data to assess the urban thermal environment in the city of Fuzhou, China. The objective of this study was to estimate the anthropogenic heat discharge in the form of sensible heat flux in complex urban environments. In order to increase the accuracy of the anthropogenic heat flux analysis, sub-pixel fractional vegetation cover was calculated by linear spectral unmixing. The result was then used to estimate latent heat flux in urban areas and to separate anthropogenic heat discharge from heat radiation due to insolation. Spatial and temporal distributions of anthropogenic heat flux were analysed as a function of land cover type, percent impervious surface area and fractional vegetation cover. The accuracy of heat fluxes was assessed using the ratios of $H, L E$ and $G$ to $R_{n}$, which were compared to the results from other studies. It is apparent that the contribution of anthropogenic heat is smaller in suburban areas, and larger in high density urban areas. However, seasonal disparities of anthropogenic heat discharge are small, and the variance of anthropogenic heat discharge is influenced by urban expansion, land cover change and increasing energy consumption. The results suggest that anthropogenic heat release probably plays a significant role in the urban heat island effect, and must be considered in urban climate change adaptation strategies. Remote sensing can play a role in mapping the spatial and temporal patterns of urban heat islands and can differentiate the anthropogenic from the solar radiative fluxes. The findings presented here have important implications for urban development planning.
\end{abstract}

Keywords: Urban; Climate change adaptation; Anthropogenic heat discharge; Sensible heat flux; Fractional vegetation cover; Percent impervious surface area; Remote sensing

\footnotetext{
* Corresponding author. Tel.: +86 5918346 5214; fax: +86 59183465397.

E-mail address: zhangyoushui@sina.com (Y.S. Zhang), hb91@leicester.ac.uk (Heiko Balzter).
} 


\section{Introduction}

Urban heat island (UHI) research usually focuses on differential air or land surface temperature in urban and rural areas. However, since anthropogenic heat released by human activities is one of the most important causes of the UHI, there is a growing body of research concerned with the impact of anthropogenic heat releases upon the urban boundary layer structure. The UHI is of particular concern because it has the potential to amplify climate change impacts on people living in cities. The UHI occurs as a result of a combination of anthropogenic heat discharge, decreased vegetation cover and increased use of artificial impervious surface materials such as concrete and asphalt (Kato and Yamaguchi 2005, Amiri and Weng 2009). These factors modify the heat balance at the land surface and eventually lead to differences in atmospheric temperature between the city and the countryside. The contributions of these factors to the thermal environment need to be clarified in order to mitigate the UHI effect and adapt effectively to climate change impacts in urban environments.

Analyses of surface energy flux have extensively been conducted over vegetated and agricultural areas, but the methods have also been applied to urban areas (Zhang et al. 1998, Chrysoulakis 2003). In urban areas, in addition to the incoming net radiation, the anthropogenic heat discharge also causes heat fluxes. Anthropogenic heat emissions include energy released by combustion of fuels and electric heat, e.g. in industry, commerce, residence, traffic, and energy losses from heating and air conditioning. The anthropogenic heat discharge occurs as ground heat, sensible heat and latent heat. The UHI effect is in part a result of increased sensible heat flux from the land surface to the atmosphere near cities. Sensible heat flux is comprised of emitted anthropogenic heat and heat radiation due to solar input. Oke (1988) suggested that the annual average anthropogenic heat release ranges from 20 to $160 \mathrm{~W} / \mathrm{m}^{2}$ for large cities. Kimura and Takahashi (1991), Klysik (1996), Grimmond (1992) and Grimmond et al. (2004) found that the average anthropogenic heat release over a city is usually less than $100 \mathrm{~W} / \mathrm{m}^{2}$, but Ichinose et al. (1999) reported $200 \mathrm{~W} / \mathrm{m}^{2}$ in the summer and $400 \mathrm{~W} / \mathrm{m}^{2}$ in the winter with a maximum of $1,590 \mathrm{~W} / \mathrm{m}^{2}$ in Tokyo. The anthropogenic component is considerably smaller than the solar component and is difficult to calculate, however its effect is not insignificant (Ichinose et al. 1999, Sailor and Lu 2004). In order to reduce current uncertainties in our quantitative knowledge of the UHI, there is a need to analyze the contribution of anthropogenic heat to the sensible heat flux, the urban thermal 
To quantify the urban surface energy balance, in situ measurements can be obtained to estimate heat fluxes accurately using spectroradiometers. However, because urban surfaces are complex, the distribution of the surface heat flux is not homogeneous. Therefore, it is extremely difficult and expensive to investigate the detailed spatial pattern of energy fluxes in a city for such measurements, if cost, time, instrument and data calibrations are considered altogether (Oke 1988). Several experimental studies were conducted in European cities, but they did not analyze the different components of the surface energy balance specifically (Piringer et al. 2002). Remote sensing data have great potential for studying the urban surface energy budget, as well as the spatial pattern and temporal dynamics of urban heat fluxes (Weng 2009). Zhang et al. (1998) used Landsat TM data, in conjunction with routine meteorological data and field measurements, to estimate the urban surface energy fluxes and analyze their spatial variability in Osaka, Japan. Chrysoulakis (2003) used ASTER imagery, together with in situ spatial data, to determine the spatial distribution of the surface net radiation balance in Athens, Greece. Kato and Yamaguchi (2005) combined ASTER and Landsat ETM+ data with ground meteorological data to investigate the spatial patterns of surface energy fluxes in Nagoya, Japan, by separating anthropogenic heat discharge and natural heat radiation from sensible heat flux. In these methods, heat fluxes were inferred using not only remote sensing data, but also locally observed meteorological data. The net increase in sensible heat flux was obtained as the residual of the heat balance equation, and the latent heat flux was assumed to be zero over impervious surfaces. The assumption that the latent heat flux was zero over impervious surfaces caused an underestimation of latent heat flux and overestimation of the net increase in sensible heat flux because the impervious surface was identified using a 'crisp' classification, in which a pixel is allocated to one class only. However, in reality many pixels represent a mixture of more than one land cover class at the spatial resolution of ASTER and Landsat ETM+.

In our study, based on a heat-balance model using satellite remote sensing and ground meteorological data, sub-pixel vegetation abundance such as fractional vegetation cover (FVC) will be used and improved to calculate latent heat flux and help to solve the issue of overestimation of the anthropogenic heat discharge from sensible heat flux. Therefore, we can estimate the artificial increase in the sensible heat flux with a higher accuracy and its contribution to urban thermal environment. The spatial distributions and temporal variations of anthropogenic heat discharge in urban thermal patterns will be further described in a quantitative manner. 


\section{Study area and data sets}

\subsection{Study area}

The study area is Fuzhou, the capital city of Fujian province, located on the southeast coast of China (Fig. 1). With a population of over 6 million, the city is situated in a subtropical plain between the Gu and Qi mountains with potential for expansion in all directions. Like many other Chinese cities, the population of Fuzhou is rapidly increasing leading to urban expansion. The urban sprawl occurs through natural surfaces being sealed by asphalt, concrete, brick tiles and metal plate surfaces for roads, buildings and other structures. Changes in land cover contribute to changes in the land surface characteristics and ecosystems. Materials such as concrete and asphalt used in urban areas have significantly different thermal properties (heat capacity/thermal inertia) and surface radiative properties (albedo and emissivity) from the surrounding rural areas.

Fig. 1 Location map of study area indicating the coverage of the Landsat TM/ETM+ images.

\subsection{Remote sensing and ground meteorological data}

A Landsat 5 TM image (acquired on June 15, 1989) and a Landsat 7 ETM+ image (acquired on March 4, 2001) were collected and processed for this study. Imagery from Landsat TM and ETM+ images has a nominal pixel resolution of $30 \mathrm{~m}$ in the shortwave bands and $60 \mathrm{~m}$ (ETM+ image) or $120 \mathrm{~m}$ (TM image) in the thermal band. The images were geometrically corrected to quality level $1 \mathrm{G}$ before delivery. Using the radiometric correction method of the Second Simulation of the Satellite Signal in the Solar Spectrum (6S), the original digital numbers of bands $1-5$ and 7 were converted to at-satellite radiance, and further converted to surface reflectance and Normalized Difference Vegetation Index (NDVI). Radiometric correction followed a two-step process. The first step was radiometric calibration, which calibrated digital numbers to at-satellite reflectance (Schroeder et al. 2006). The second step was the calculation of land surface reflectance using the radiative transfer model 6S. The digital numbers of the thermal band (band 6) were converted to radiation luminance or top-of-atmospheric radiance, then the atmospheric correction tool MODTRAN 4.1 was applied to estimate three parameters: atmospheric transmission, upwelling and downwelling radiance. These enable the calculation of the surface-leaving radiance by removing atmospheric effects (Yuan and Bauer 2007). Lastly, the surface-leaving radiance is converted to land surface temperature (LST) according to Chander and Markham (2003) and 
1 Zhang et al. (2009) for TM/ETM+ data. The land surface emissivity was based on the landcover classification by Yuan and Bauer (2007) or the emissivity values as derived by Snyder et al. (1998).

The visible and near infrared data were used to classify the land cover type. A maximum likelihood classification method was carried out to extract seven land cover types in the study area, namely: forest, water, agriculture, bare land, grassland, suburban and urban (Fig. 2).

Fig. 2 Land cover types of the study area derived from the TM image acquired in 1989 (left) and ETM+ image acquired in 2001 (right).

In the present study, the ground meteorological data used were solar radiation, wind speed, relative humidity, air pressure and air temperature at the acquisition time of the $\mathrm{TM} / \mathrm{ETM}+$ scenes. The meteorological data were recorded at local meteorological stations. Solar radiation, wind speed and relative humidity were assumed to be constant throughout the study area. Because the research area is not large, meteorological data from one station located near the central part of the image can reasonably represent the whole study area. Water vapour pressure was calculated from the relative humidity and saturation water vapour pressure, which was obtained from the atmospheric temperature (Brutsaert 1982). Table 1 shows the meteorological data measured close to the times of the satellite overpasses in Fuzhou.

Table 1 Meteorological parameters used to calculate heat fluxes in Fuzhou

\section{Methods of heat flux calculation}

3.1 Net radiation, ground and sensible heat fluxes calculation

Calculation theories of net radiation, sensible heat, latent heat and ground heat fluxes have already been established. For natural land surfaces, the surface energy balance is commonly written as:

$$
R_{n}=G+H+L E
$$

30 Where $R_{n}$ is the net radiation, $G$ is the ground heat flux, $H$ is the sensible heat flux, and $L E$ is 31 the latent heat flux.

Anthropogenic heat discharge is the sum of heat released by industry, vehicles and living activities. In urban areas, the energy consumption due to human activities generates 
1 anthropogenic heat discharge in the forms of sensible heat, ground heat and latent heat. The heat balance can hence be modified as follows:

$$
R_{n}+A=G+H+L E
$$

where $A$ is the anthropogenic heat discharge released by the energy consumption due to human activities. Therefore, $A$ is mainly transferred from urban areas to heat the atmosphere directly and contributes to increase the surface temperature of buildings, other structures and the ground. Therefore, $A$ significantly alters the extent and magnitude of the UHI. Surface temperature and heat fluxes measured by remote sensing are affected by anthropogenic heat discharges. Based on the land surface energy balance, the effect of anthropogenic heat discharge on sensible heat flux can be calculated.

The equation to calculate the net radiation is given by

$$
R_{n}=(1-a) \cdot R_{s w d}+\varepsilon \cdot R_{l w d}-\varepsilon \cdot \sigma \cdot T_{0}^{4}
$$

where $a$ is the albedo related to land surface types, $R_{s w d}$ is the downward solar radiation, $R_{l w d}$ is the downward longwave radiation, $\varepsilon$ is the emissivity of the surface, $\sigma$ is the StefanBolzmann constant, and $T_{0}$ is the surface temperature. $a, \varepsilon$ and $T_{0}$ can be derived from remote sensing data. The downward solar radiation $R_{s w d}$ can be calculated by the equation:

$$
R_{s w d}=I_{s c} \cdot e_{0} \cdot \cos \theta_{\mathrm{z}} \cdot \exp (-\mathrm{m} \cdot \tau)
$$

where $I_{s c}=1367 \mathrm{~W} / \mathrm{m}^{2}$ is the solar constant, $e_{0}$ the eccentricity factor, $\theta_{\mathrm{z}}$ the solar zenith angle, $m$ the air mass, and $\tau$ the optical thickness. Details on the determination of all the parameters can be found in Iqbal (1983). When there is no measurement, the downward longwave radiation $R_{l w d}$ can be calculated as:

$$
R_{l w d}=\varepsilon_{a} \cdot \sigma \cdot T_{a}^{4}
$$

where $\varepsilon_{a}$ is the emissivity of the atmosphere which can be estimated using the Swinbank formula as given by Campbell and Norman (1998) in the form $\varepsilon_{a}=1.24 *\left(e_{a} / \mathrm{T}_{\mathrm{a}}\right)^{1 / 7}$, with $e_{a}$ is the atmospheric water vapor pressure in $\mathrm{hPa}$ and $T_{a}$ the air temperature at the reference height.

The magnitude of ground heat flux $G$ is generally small, and the determination of this flux in energy-balance studies is often handled by parameterising it as a constant fraction of the net radiation. However, for specific surfaces such as bare soil, non-natural materials or urban surfaces in this study, $G$ can roughly be estimated from the net radiation:

$$
G=c_{g} \cdot R_{n}
$$

31 Here, the coefficient $c_{g}$ is fixed according to surface type and season by referring to actual measurements, the $c_{g}$ values of all surface types in the present study are based on Brutsaert (1982), Anandakumar (1999), Silberstein et al. (2001) and Kato and Yamaguchi (2005). 
Bastiaanssen (2000) defined sensible heat flux $H$ as

$$
H=\rho_{a} \cdot c_{p} . \delta T / r_{a h}
$$

3 Where $\rho_{a}$ is the air density $\left(\mathrm{kg} / \mathrm{m}^{3}\right), c_{p}$ is corrected based on air pressure and vapour pressure in order to adjust for atmospheric humidity, $\delta T$ the surface-air temperature difference calculated as below and $r_{a h}$ the aerodynamic resistance $(\mathrm{s} / \mathrm{m})$ to heat transport. $r_{a h}$ is calculated as (Choudhury et al. 1986):

$$
r_{a h}=\left\{\ln \left[(Z-d) / Z_{o m}-\psi\right] \cdot \ln \left[(Z-d) / Z_{o h}-\psi\right]\right\} /\left(k^{2} u_{z}\right)
$$

where $Z$ is the height $(\mathrm{m} / \mathrm{s})$ at which the wind speed $u_{z}$ (at height $\mathrm{z}$ ) is measured; $d$ the zero plane displacement height; $Z_{o m}$ and $Z_{o h}$ are respectively the roughness lengths for momentum and heat transport. $\psi$ is the stability correction function, which depends on the MoninObukhov length $L$ (Brutsaert 1982), and $k$ is von Karman's constant (0.4). $\psi$ is calculated as described by Choudhury et al. (1986). Typical values of roughness lengths, $Z_{o m}$ and $Z_{o h}$, for surface types are alternatively used in this study (Brutsaert 1982, Choudhury et al. 1986, Kondo 1994, 2000, Hansen 1993, Yasuda 1995, Kato and Yamaguchi 2005, 2007). The surface types were obtained from image classification with the Maximum Likelihood method. Several empirical equations for the estimate of $d, Z_{o m}$ and $Z_{o h}$ have been developed. Based on Pielke et al. (1983), Stull (1988) and Kato and Yamaguchi (2005), the zero plane displacement height and roughness lengths $\left(Z_{o m}=10 Z_{o h}\right)$ used in this study are listed in Table 2 .

Table 2 Zero plane displacement height $\mathrm{d}$ and roughness lengths $Z_{o m}$ and $Z_{\text {oh }}$ for land cover types in Fuzhou

$H$ can be estimated inferring surface temperature $T_{0}$ from remote sensing and air temperature $T_{a}$ from synoptic measurements. Kalma and Jupp (1990) and Gay and Bernhofer (1991) conducted measurements over wet surfaces under arid conditions, which showed that $T_{a}$ can exceed $T_{0}$ by several degrees during daytime. As $T_{0}$ and $T_{a}$ for each land unit needs to be measured at different elevations and exactly on the same moment and place with a high precision, the combination of $T_{0}$ and $T_{a}$ measurements to estimate $\delta T$ have severe practical problems (Bastiaanssen et al. 1998). Therefore, it can be easier to estimate $\delta T$ (K) from an inversion of the transfer equation for sensible heat.

$$
\delta T=T_{0}-T_{a}=H \cdot r_{a h} /\left(\rho_{a} c_{p}\right)
$$

In Eq. (9), $H$ is only known at the extremes of $T_{0}$, i.e. for $H=0$ (for the coldest pixels) and $H$ $33=R_{n}-G$ (for the warmest pixels). For these specific land surface elements, $\delta T$ can be 
1 with high-thermal-emission surfaces. Therefore, $T_{0}$ may be used to interpret $\delta T$ across the image. For heterogeneous land surfaces, the following regression equation is assumed to be valid:

$$
\delta T=\mathrm{a} \cdot T_{0}-\mathrm{b}
$$

where $a$ and $b$ are the linear regression coefficients valid for one particular moment in time and a specific landscape. The correctness of Eq. (10) has been demonstrated using data collected from large scale field experiments in many study areas (e.g. Wang et al. 1995, Franks and Beven 1997, Bastiaanssen et al. 1998). In our study, the minimum and maximum values of $\delta T$ can be calculated for the coldest and warmest pixels, and $a$ and $b$ are calculated based on these pixels with given $H, \delta T$ and $T_{0}$ values. Then, we can calculated $H$ for the whole image.

3.2 Latent heat flux calculation with FVC in urban area

According to Monteith and Unsworth (1990) latent heat flux is expressed as:

$$
L E=\rho_{a} c_{p}\left(e_{s}-e_{a}\right) / \gamma\left(r_{a}+r_{s}\right)
$$

where $e_{s}$ is the saturation water vapour pressure in $\mathrm{hPa}$ at the evaporating surface temperature, $e_{a}$ is the atmospheric water vapour pressure in $\mathrm{hPa}, \gamma$ is the psychrometric constant in $\mathrm{hPa} / \mathrm{K}^{1}$, and $r_{s}$ is the stomatal resistance in $\mathrm{s} / \mathrm{m}$ which describes the resistance of vapour flow through the transpiring vegetation and evaporating soil surface. Stomatal resistance is calculated based on the Jarvis-type scheme simplified by Nishida et al. (2003). Jarvis (1976) and Nishida et al. (2003) assume that the environmental factors, namely air temperature, vapour pressure deficit (VPD), photosynthetically active radiation (PAR), soil water potential, and atmospheric $\mathrm{CO}_{2}$ concentration control stomatal conductance in the following form:

$$
1 / r_{s}=f_{1}\left(T_{\mathrm{a}}\right) f_{2}(\mathrm{PAR}) f_{3}(\mathrm{VPD}) f_{4}(\psi) f_{5}\left(C O_{2}\right) / r_{\text {sMIN }}+1 / r_{\text {cuticle }}
$$

Where $\psi$ is the leaf-water potential $(\mathrm{Pa}), r_{s M I N}$ the minimum resistance $(\mathrm{s} / \mathrm{m})$, and $r_{\text {cuticle }}$ the canopy resistance related to diffusion through cuticle layer of leaves (s/m). In Eq. (12), air temperature $T_{a}$ and $P A R$ can be estimated from satellite data and radiative transfer equations. $V P D$ and $\psi$ are hard to estimate from satellite data. Some studies have pointed out that some parameters could sometimes act as surrogates for others in Eq. (12) (Hipps et al. 1996, Tanaka et al. 2000, Toda et al. 2000). Therefore, we dropped the terms of $V P D, \psi$ and $\mathrm{CO}_{2}\left(f_{3}\right.$, $f_{4}$ and $f_{5}$ ) from Eq. (12) and used a simplified equation in our study. $f_{1}, f_{2}, r_{s M I N}$ and $r_{\text {cuticle }}$ are estimated by the equations proposed by Javis (1976), Kelliher et al. (1995), Kosugi (1996) and White et al. (2000). 
The latent heat is the energy used for the transport of water vapour from the land surface, through vegetation evapotranspiration or evaporation from the soil to the atmosphere (Monteith and Unsworth 1990). The LE from impervious surfaces estimated by remote sensing data was usually assumed zero. Impervious surfaces are often identified using crisp (as opposed to fuzzy) classification schemes, in which a pixel is allocated to one class only. However, many pixels represent a mixture of more than one land cover type at the spatial scale of ASTER and Landsat TM/ETM+, and the effect of mixed pixels containing mostly but not exclusively sealed impervious surfaces has led to an underestimation of latent heat fluxes in urban areas in other studies. In the study presented here, linear spectral unmixing (LSU) is used to extract FVC at a sub-pixel level. $L E$ is then calculated in proportion to fractional vegetation cover in every image pixel of urban areas, and only set to zero when the surface type was classified as $100 \%$ impervious surface area (ISA), based on the assumption that artificial land surfaces were dry at the times when the satellite images were acquired.

3.3 Estimation of anthropogenic heat flux in urban area

In Eq. (3), the net radiation mainly depends on land surface types and atmospheric temperatures in the long-wave part of the spectrum. It is affected relatively little by changes in air temperature, because the amount of short-wave radiation is much greater than the net longwave radiation under clear sky conditions. In Eq. (7), $H$, calculated based on observed surface and air temperatures, is influenced by the radiant heat balance and the effect of the anthropogenic heat discharge. In Eq. (11), $L E$ is only due to evapotranspiration. Therefore, temperature change mainly impacts on $H$ and the surplus in the radiant heat balance due to the anthropogenic effect on the urban surface. Its influence on $L E, R_{n}$ and $G$ is negligible.

The sensible heat flux due to the radiant heat balance $\left(H_{n}\right)$ can be calculated as the residual from the heat-balance equation:

$$
H_{n}=R_{n}-G-L E
$$

27 The sensible heat flux due to the artificial effects $H_{a s}$ can be calculated as the difference between the total sensible heat flux $H$ in Eq. (7) and $H_{n}$ as follows:

$$
H_{a s}=H-H_{n}
$$

When $H$ is greater than or equal to $H_{n}$, we can use Eq. (14) to calculate $H_{a s}$, otherwise $H_{a s}$ is 0 .

\section{Results and discussion}


Based on the assumption that impervious surface areas were dry at the time of image acquisitions, a zero latent heat flux was assumed when the surface type is urban, suburban or industrial. In our study, fractional vegetation cover (FVC, 0-100\%) was used to improve the calculation of $L E$ in those surface types.

FVC was used to solve the issue of $L E$ underestimation in urban areas by quantitatively analyzing the relationship between FVC and $L E$. Fig. 3a and b show the scatterplots of $L E$ and FVC for a sample of pixels with FVC $>18 \%$ in urban and peri-urban areas. The results indicate a statistical correlation between $L E$ and $\mathrm{FVC}(\mathrm{P}<0.05 ; \mathrm{r}=0.704$ for 1989 and $\mathrm{r}=0.771$ for 2001). To further investigate these relationships, a zonal analysis was carried out to evaluate the mean $L E$ at increments of FVC from $18 \%$ to $100 \%$. Fig. 3c and d are showing scatterplots of mean $L E$ and corresponding FVC. The results indicate a statistically significant correlation ( $\mathrm{P}<0.05 ; \mathrm{r}=0.954$ in 1989 and $\mathrm{r}=0.967$ in 2001) between mean $L E$ and FVC. Here, the LSU method was used to extract FVC at a sub-pixel level. Because of the limitation of this method and the complexity of the spectrum of mixed pixels in an urban landscape, we could not accurately estimate FVC of less than 18\%. It is well known that the predominantly bare ground surfaces tend to exhibit larger variation in surface radiant temperature than the densely vegetated LULC types. Therefore, relationships between $L E$ and FVC in the conditions of $\mathrm{FVC}<18 \%$ were not analyzed.

Fig. 3 Scatterplots of $L E$ versus FVC (a and b) and linear plots of mean $L E$ versus FVC (c and d) in urban area

The strong linear relationships between mean $L E$ and FVC for both 1989 and 2001 suggest that the variations in $L E$ can be well accounted for by FVC during the summer and spring period. The resulting regression equations of mean $L E$ and FVC were used to correct $L E$ in partially vegetated urban areas that are characterised by mixed pixels. $L E$ in urban areas with FVC <18\% was assumed to be zero. Fig. 4a and b show maps of $L E$ for June 15, 1989 and March 4, 2001, respectively, in which $L E$ was assumed zero for all impervious surfaces based on a crisp classification. Fig. $4 \mathrm{c}$ and d show maps of $L E$ that are estimated using subpixel FVC in urban and suburban areas based on spectral unmixing. It is obvious that FVC, 
1 a crisp classification ignores within-pixel heterogeneity, $L E$ in urban and suburban areas as 2 shown in Fig. 4a and $\mathrm{b}$ was underestimated because this method neglects mixed pixels that are partially vegetation urban areas. Comparing the spatial distributions of $L E$ in Fig. 4a and c with Fig. $4 \mathrm{~b}$ and $\mathrm{d}$ respectively, the contrast between $L E$ in urban and surrounding areas was stronger in 2001 (Fig. 4b and d) than in 1989 (Fig. 4a and c). This pattern is more pronounced in 2001 than in 1989 because urban planners took landscape planning and green spaces in urban and suburban areas into account along with the urban expansion. crisp classification method; image acquired on March 4, 2001; (c) $L E$ for impervious surfaces corrected using FVC from spectral unmixing; image acquired on June 15, 1989; (d) $L E$ for impervious surfaces corrected using FVC from spectral unmixing; image acquired on March 4, sensible heat flux. Each flux was calculated respectively by Eqs. (3), (6), (7), (11) and (13). Anthropogenic heat discharge from $H$ on June 15, 1989 and March 4, 2001 calculated by Eq. (14) is shown in Fig. 5. In Fig. 5, $H_{a s}$ was high in urban areas and low in natural surfaces for two seasons. Compared with Fig. 2, it is obvious that $H_{a s}$, calculated as a difference between $H$ and $H_{n}$, of vegetation, water and bare soil areas has negative values. Theoretically, $H_{a s}$ for natural surfaces should be 0 . The reason for these negative values can be regarded as estimation errors for each flux calculated by Eqs. (3), (6), (7), (11) and (13). The aim of this research was to analyze $H_{a s}$ in urban areas and hence the parameters of surface meteorological measurements in Table 1 are only observed in urban areas, causing errors in the $H_{a s}$ estimation for non-urban surface types. Because the urban areas expanded significantly between 1989 and 2001, this trend implies that the results of the spatial pattern of $H_{a s}$ are reasonable estimates of the real spatial pattern. It also indicates that the magnitude of $H_{a s}$ can 
1 calculation in urban areas are available.

Fig. 5 Anthropogenic heat discharge from sensible heat flux on (a) June 15, 1989 and (b)

March 4, 2001

4.2 Spatial-temporal distributions of anthropogenic heat flux

In order to analyze $H, H_{a s}, L E$ and $G$ in different land cover categories in urban and peripheral areas, the mean and standard deviation (SD) of heat fluxes for each surface type are shown in Table 3. The urban and suburban areas have significantly higher mean $H_{a s}$ values than other land cover types, but the standard deviation of $H_{a s}$ is lower than for other surface types both in 1989 and 2001 . Hence, we can conclude that the accuracy of $H_{a s}$ for urban and suburban areas is higher than for other land cover types. In the central area, the daytime anthropogenic heat release in developed areas is large, indicating heat release characteristics occurring when offices and commercial facilities are active. In Table 3, the mean $H_{a s}$ is higher in the urban areas than the peripheral areas, which indicates that the calculated $H_{a s}$ is a realistic estimate.

In urban areas, the mean $H_{a s}\left(50.2 \mathrm{~W} / \mathrm{m}^{2}\right)$ on June 15,1989 was lower than the estimate of $94.4 \mathrm{~W} / \mathrm{m}^{2}$ on March 4, 2001. The main reason is the increased energy consumption as a result of anthropogenic activities between 1989 and 2001. For Fuzhou city, energy consumption variations are not large between early summer and spring of the same year. However, the heat emissions from buildings, traffic and other factors have increased markedly from 1989 to 2001 because of urban expansion and population growth. The heterogeneity of the suburban landscape is more complex than that in urban areas. The SD of $H_{a s}$ in suburban areas is higher than in urban areas in both years. If correlated parameters such as aerodynamic resistance to $H$, stomatal resistance to $L E$ and parameters such as wind speed, roughness length, displacement height, FVC and land cover type etc. can be accurately quantified, the $H_{a s}$ in this area can be estimated accurately. 
In Table 3, $H$ is significantly higher for urban and suburban areas than for other land cover types. This relationship is stable for both seasons. Because of seasonal and solar radiative differences, the surface temperature rise caused by incoming solar radiation was higher in summer than in spring, so $H$ is usually higher in summer than that in spring. In this study, $H$ of forest, water, grassland and suburban was higher in 1989 than in 2001, while $H$ of water bodies had similar values in both years. However, for agriculture, bare land and urban areas, $H$ values were higher in 2001 than in 1989. The main reasons for this increase are related to agricultural activities, soil moisture and the seasonal variation of the meteorological parameters used in the estimation of $\mathrm{H}$.

Mean $L E$ on forest, water, agriculture and grassland is high in both seasons, and mean $L E$ of these land cover types was higher in 2001 than in 1989 because the meteorological parameters were different. The wind speed in 2001 was much higher $(9 \mathrm{~m} / \mathrm{s})$, and the relative humidity was significantly lower (23\%) than in $1989(1 \mathrm{~m} / \mathrm{s}$ and 52\%, respectively). These meteorological parameters impact greatly on $L E$. However, for bare land, suburban and urban areas with low water content, mean $L E$ was slightly higher in 1989 than in 2001 due to incoming solar radiation differences between summer and spring.

Mean values of $G$ of urban and suburban areas in summer were significantly larger than in spring, and the values of $G$ of urban and suburban areas were larger than those of other land cover types. $G$ of water is 0 . The urban surface stores more heat than the surrounding low building and impervious area during the daytime. For forest, bare land, grassland, suburban and urban land cover types, mean $G$ was larger in 1989 than in 2001, but not for water and agriculture.

Theoretically, $H_{a s}$ should be $0 \mathrm{~W} / \mathrm{m}^{2}$ for natural surfaces such as bare soil, grass and forest areas. In Table 3, the mean and standard deviation of $H_{a s}$ of forest, water, agriculture and grassland was all negative in both seasons, illustrating an estimation error. The main reasons for this error are that the accuracy of the calculation of the aerodynamic resistance to $H$, stomatal resistance to $L E$ and correlated parameters to $G$ in these areas are not high. Also some parameters such as wind speed, roughness lengths, displacement height, FVC and land cover types etc., need to be estimated further in order to adjust to the actual surface conditions for more accurate calculation of $H$ and $L E$, and $G$.

We created several imperviousness categories for further analysis. The percent ISA was estimated by LSU within a continuous range of between $0 \%$ and $100 \%$. Pixels were classified as urban when the percent ISA was $\geq 10 \%$. Water was excluded from the classification. The 
1 density; 30-50\% for medium density; and $>50 \%$ for high-density. High density urban area was further divided in three levels: $50-70 \%, 70-90 \%$ and $>90 \%$. The mean and SD of $H$ and $H_{a s}$ for each imperviousness category in urban and suburban areas are shown in Table 4. The high-density urban area has higher means for $H$ and $H_{a s}$ than those in other zones for 1989 and 2001. $H$ and $H_{a s}$ for medium and high-density urban areas was higher in 2001 than in 1989. Comparatively, $H_{a s}$ of high-density urban development areas was on average 103.5 $\mathrm{W} / \mathrm{m}^{2}$ in 2001 and only $42.2 \mathrm{~W} / \mathrm{m}^{2}$ in 1989 . The main reason for this increase over time is probably that the heat emission from buildings and traffic (energy consumption by human activities) increased significantly, urban development expanded from 1989 to 2001, and seasonal and climatic fluctuations occurred. Landscape heterogeneity increases from low and medium density urban to high-density urban areas, which indicates that the urban landscapes would have experienced wider variation in $H$ than the natural vegetation because of the mix of LULC types, different building structures and construction materials. This explains the thermal heterogeneity that characterizes these areas. Generally, the SDs of $H$ and $H_{a s}$ also show this pattern. As ISA increases from low to high urban development density, vegetation cover gradually decreases with concomitant increase in $H$ and $H_{a s}$. Vegetation keeps the temperature low by transpiration, so that $L E$ is high and $H$ is low in vegetation areas. Therefore, the natural vegetation canopy in urban area reduces the surface radiant temperature leading to relatively low $H$ and $H_{a s}$ values.

Table 4 Mean $H$ and $H_{a s}$ of percent ISA categories and associated standard deviation (SD) in urban and suburban areas in 1989 and $2001 \quad\left(\mathrm{~W} / \mathrm{m}^{2}\right)$

Fig. 6 is the frequency distribution of $H_{a s}$ in medium and high density urban areas. Fig. 6a shows that $H_{a s}$ on June 15,1989 , with about 0 to $80 \mathrm{~W} / \mathrm{m}^{2}$, has a higher frequency. However, Fig. 6b shows the frequency distribution of $H_{a s}$ on March 4, 2001. $H_{a s}$ values between 200 and $250 \mathrm{~W} / \mathrm{m}^{2}$ and less than $50 \mathrm{~W} / \mathrm{m}^{2}$ have higher frequencies. It is evident that anthropogenic heat release in the urban area of Fuzhou is concentrated in different ranges between early summer 1989 and early spring 2001. Anthropogenic heating and the magnitude and extent of the anthropogenically forced heat island was greater in 2001 than in 1989. The reason of this phenomenon is mainly attributed to urban expansion and energy consumption change due to human activities in buildings, factories and residences and transportation for 1989 and 2001. The seasonal disparities of $H_{a s}$ in study area are small. The energy usage of Fuzhou for human use grew between 1989 and 2001, so this result is plausible. 
(b) March 4, 2001

Fig. 6 Frequency distribution of anthropogenic heat flux of the medium- and high- density urban areas

6

To further investigate the relationship between heat fluxes and surface sealing, Fig. 7 depicts the plots of mean $H, H_{a s}$ and FVC for each ISA category in urban and sub-urban areas for 1989 and 2001. In both cases (Fig. 7a and b) high average FVC (>90\%) is shown to have lowered the mean $H$ by more than $200 \mathrm{~W} / \mathrm{m}^{2}$ in 1989 and $300 \mathrm{~W} / \mathrm{m}^{2}$ in 2001 . In high density urban areas, compared with completely sealed urban areas with 100\% ISA, FVC (50\%) lowered mean $H_{a s}$ by about $80 \mathrm{~W} / \mathrm{m}^{2}$ in 1989 and by slightly more than $100 \mathrm{~W} / \mathrm{m}^{2}$ in 2001 . Dense natural vegetation canopies in urban areas reduce the surface radiant temperature leading to relatively low $H$ and $H_{a s}$ values. As ISA increases from medium to high urban development density, mean FVC decreases with concomitant gradual increase in $H$ and $H_{a s}$. Fig. 7a and b show a negative relationship between $H$ and FVC, especially if ISA $>90 \%$. The relationship between $H_{a s}$ and FVC is also negative but not significant in high density urban areas (ISA from $50 \%$ to $90 \%$ ), but it is significant if ISA $>90 \%$. These findings provide important information for urban planners to adapt to climate change impacts in UHIs.
(a) June 15, 1989
(b) March 4, 2001

Fig. 7 The 1989 and 2001 mean $H, H_{a s}$ and FVC for each percent ISA category in urban and sub-urban areas.

Fig. 7 shows that $H_{a s}$ and $H$ in high density urban areas are positively correlated. This correlation is hardly affected at all by the season. Fig. 7 also shows the temporal and seasonal variations and patterns of $H_{a s}$ in Fuzhou in 1989 and 2001. In the subtropical climate of Fuzhou, the seasonal disparities of $H_{a s}$ between early spring and early summer energy consumption vary little. Anthropogenic heating is influenced by urban expansion and lifestyle changes with implications for energy consumption.

\subsection{Accuracy analysis and validation of heat fluxes}

Though solar radiation in spring is less than in summer, energy consumption due to urban expansion and human activities in 2001 was higher than in 1989. The mean $H_{a s}$ of 
1 urban areas, especially high density urban areas, on June 15, 1989 was lower than that on March 4, 2001. These results show that the $H_{a s}$ calculation gives plausible results.

$H_{a s}$ is calculated as the surplus in the radiant heat balance, therefore the calculations of $H$, $L E, G$ and $R_{n}$ influence the accuracy of $H_{a s}$. It is apparent that there are some errors in the calculation of $H_{a s}$ for natural surfaces. In the calculation of $H$, there were also some errors in calculating surface-air temperature difference $(\delta T)$, aerodynamic resistance $\left(r_{a h}\right)$, roughness lengths $\left(\mathrm{Z}_{\mathrm{om}}\right.$ and $\left.\mathrm{Z}_{\mathrm{oh}}\right)$ and the zero plane displacement height $(d)$. The $L E$ calculation also includes errors, though we used FVC to improve estimates of $L E$, errors also arise from calculating the aerodynamic resistance and the stomatal resistance. Especially the influence of the roughness lengths on $H$ and $L E$ calculations is considerably large. The calculation of $G$ and $R_{n}$ also contained errors, and the errors varied with season.

Because the $H_{a s}$ estimation was affected by these errors, it became necessary to validate the resultant heat fluxes. Since in situ measurements of $H_{a s}$ and other heat fluxes were not available, we compared the results in the present study with previously published studies by others in similar conditions (Christen and Vogt 2004, Grimmond et al.2004, Moriwaki and Kanda 2004, Kato and Yamaguchi 2005, 2007, Offerle et al. 2005). Heat fluxes are influenced by seasonal variation, therefore any comparison of heat flux values is limited by the site and seasonal differences. Table 5 shows the relative ratios of heat fluxes $H, L E$ and $G$ to the net radiation $R_{n}$ for these measurements in the daytime. The heat fluxes used in the present study and in Kato and Yamaguchi $(2005,2007)$ were averaged over $3 * 3$ pixels and $5 * 5$ pixels respectively, others were in situ measurement data.

Table 5 Ratios of heat fluxes to the net radiation of the present study and measured previously in spring are lower than those published by Grimmond et al. (2004) and similar to other measurements except the summer data by Kato and Yamaguchi (2007) in Table 5. The main reason of the minor difference is thought to be the difference in incoming solar radiation and surface characteristics. Kato and Yamaguchi (2007) assumed $L E$ to be zero over impervious surfaces and therefore may have underestimated $L E$ in urban areas, we used LSU to estimate FVC and to improve the $L E$ calculation in urban areas. In Table 5 , the $L E / R_{n}$ ratios of 0.06 in summer and 0.04 in spring in urban area are higher than 0 given by Kato and Yamaguchi 
1 in summer and 0.57 and 0.58 in urban and sub-urban areas in spring are higher than some but 2 lower than other studies presented in Table 5. Surface type influences the value of $G$ and the seasonal pattern of $G$. However, $G / R_{n}$ varied little between spring and summer in the present study.

Because the differences are partially caused by differences in the structural and material properties of the land surface and the seasonal climate, it is difficult to compare our estimates directly with other studies as shown in Table 5. Generally, the results from the above comparisons indicate that the estimation in our study gives reasonable results that are broadly in line with previously published studies.

\section{Conclusions}

Anthropogenic heat flux $H_{a s}$ estimates are necessary for regional climate modelling, because they alter the extent and magnitude of the UHI significantly. We have shown that after the derivation of $H, L E$ and $G$, it is possible to estimate the $H_{a s}$ as a residual of the energy balance equation. Accurate estimation of anthropogenic heat emissions is very important for investigating urban micro-meteorology and human impacts on urban climates, as well as associated air pollution and human health issues such as respiratory diseases.

This study demonstrates an alternative methodology for using remote sensing to estimate the magnitude, spatial and seasonal patterns of $H_{a s}$. The method is demonstrated for the city of Fuzhou, China. In order to solve the issue that $L E$ in urban areas has often been underestimated, we suggest a method that takes into account the $L E$ of sub-pixel vegetation patches in pixels classified as impervious surfaces. Estimating FVC at the sub-pixel scale can resolve this issue and improve the accuracy of $L E$ and anthropogenic heat discharge estimates.

Anthropogenic heat is man-made heat generated by electricity inputs to buildings, fuel combustion of traffic and industrial activities. In our study, seasonal disparities of energy consumption between early spring and early summer are small. Urban expansion and land cover change due to human activities are the main impact factors on the anthropogenic heat discharge. The results show that anthropogenic heat discharge was larger in early spring 2001 than in early summer 1989.

The heat fluxes derived by the proposed method are compared with in situ measurements in the $H / R_{n}, L E / R_{n}$ and $G / R_{n}$ ratios. The results show that the calculations of $H_{a s}$ and other heat fluxes were plausible. In this study, the spatial distribution and seasonal variation of $H_{a s}$ were analyzed and compared for daytime in summer and spring. Future research should address all 
1 the seasonal and diurnal variations of $H_{a s}$ in urban areas and compare $H_{a s}$ for cities under different climatic conditions.

Efforts are still underway to improve methodologies for estimation of heat fluxes in urban areas using remote sensing data. Out of the various variables used to estimate the energy balance, the estimation of aerodynamic resistance $\left(r_{a}\right)$ to $H$ is difficult, because it is influenced by wind speed, surface roughness and displacement height and cannot be determined analytically. Currently, there has not been an effective method that can accurately estimate the surface roughness and displacement height in urban areas with spatial heterogeneity and buildings of different height. The stomatal resistance $\left(r_{s}\right)$ to latent heat transfer is also difficult to estimate precisely. Therefore, it is difficult to calculate urban anthropogenic heat release precisely.

In future studies, there is a need to differentiate the industrial heat release, domestic heat release and traffic heat release from anthropogenic heat release using ancillary data and relate these fluxes to population density. The potential contribution of anthropogenic heat release to the development of the UHI effect needs to be analyzed further for a globally representative sample of cities.

\section{References}

Amiri, R., Weng, Q., Alimohammadi, A. and S.K. Alavipanah., 2009, The spatial-temporal dynamics of land surface temperatures in relation to fractional vegetation cover and land use/cover in the Tabriz urban area, Iran. Remote Sensing of Environment, 113, pp. 2606-2617.

Anandakumar, K., 1999, A study on the partition of net radiation into heat fluxes on a dry asphalt surface. Atmospheric Environment, 33, pp. 3911-3918.

Bastiaanssen, W. G. M., Menenti, M., Feddes, R. A. and Holtslag, A.A.M., 1998, A remote sensing surface energy balance algorithm for land (SEBAL): 1. Formulation. Journal of Hydrology, 212-213, pp. 198-212.

Bastiaanssen, W. G. M., 2000, SEBAL-based sensible and latent heat fluxes in the irrigated Gediz Basin, Turkey. Journal of Hydrology, 229, pp. 87-100.

Brutsaert, W., 1982, Evaporation into the Atmosphere, Theory, History and Applications. Reidel, Dordrecht, The Netherlands.

Campbell, G. S., Norman, J. M., 1998, An Introduction to Environmental Biophysics, Springer-Verlag, New York. 286 pp. 
1 Chander, G., Markham, B., 2003, Revised Landsat-5 TM radiometric calibration procedures and postcalibration dynamic ranges. IEEE Transactions on Geoscience and Remote Sensing, 41, pp. 2674-2677.

Christen, A., Vogt, R., 2004, Energy and radiation balance of a central European city. International Journal of Climatology, 24, pp. 1395-1421.

Choudhury, B. J., Reginato, R. J., Idso, S. B., 1986, An analysis of infrared temperature observations over wheat and calculation of latent heat flux. Agric. Forest Meteorol., 37, pp. $75-88$.

Chrysoulakis, N., 2003, Estimation of the all wave urban surface radiation balance by use of ASTER multispectral imagery and in situ spatial data. Journal of Geophysical Research, 108, pp. doi: 10. 1029/2003JD003396.

Franks, S. W., Beven K. J., 1997, Bayesian estimation of uncertainty in land surfaceatmosphere flux predictions, Journal of Geophysical Research. 102(D20), pp. 23991-23999.

Gay, L. W., Bernhofer, C., 1991, Enhancement of evapotranspiration by advection in arid regions. In: Kienitzetal.(Eds.), Hydro-logical interactions between atmosphere, soil and vegetatation. Proc. Vienna conference, vol. 204. IAHS Publication, 147-156.

Grimmond, C. S. B., 1992, The suburban energy balance: methodological considerations and results for a mid latitude west coast city under winter and spring conditions. Int. J. Climatol., 12, pp. 481-497.

Grimmond, C. S. B., Salmond J. A., Oke, T. R., Offerle, B. and Lemonsu, A., 2004, Flux and turbulence measurements at a densely built-up site in Marseille: heat, mass (water and carbon dioxide), and momentum. J. Geophys. Res., 109, pp. 24101.

Hansen, S. V., 1993, Surface roughness length. ARL technical report, U. S. Army, White Sands Missile Range, NM 88002-5501.

Hipps, L. E., Or, D., Neale,C. M. U., 1996, Spatial structure and scaling of surface fluxes in a Great Basin ecosystem, in Scaling Up in Hydrology Using Remote Sensing, edited by J. B. Stewart et al., pp. 113-125, John Wiley, New York.

Ichinose, T., Shimodozono, K., Hanaki, K., 1999, Impact of anthropogenic heat on urban climate in Tokyo. Atmos. Environ., 33, pp. 3897-3909.

Iqbal, M., 1983, An introduction to solar radiation. Academic Press, Toronto.

Jarvis, P. G., 1976, The interpretation of the variations in leaf water potential and stomatal conductance found in canopies in the field. Philosophical Transactions of the Royal Society of London. Series B, Biological Sciences, 273, pp. 593-610. 
1 Kalma, J. D., Jupp, D. L. B., 1990, Estimating evaporation from pasture using infrared thermometry: evaluation of a one-layer resistance model. Agr. And Forest Meteorology, 51, pp. 223-246.

Kato, S., Yamaguchi, Y., 2005, Analysis of urban heat-island effect using ASTER and ETM+ data: Separation of anthropogenic heat discharge and natural heat radiation from sensible heat flux. Remote Sensing of Environment, 99, pp. 44-54.

Kato, S., Yamaguchi, Y., 2007, Estimation of storage heat flux in an urban area using ASTER data. Remote Sensing of Environment, 110, pp. 1-17.

Kelliher, F. M., Leuning, R., Raupach, M. R. and Schulze, E. D., 1995, Maximum conductance for evaporation from global vegetation types. Agric. For. Meteorol., 73, pp. $1-16$.

Kimura, F., Takahashi S., 1991, The effects of land-use and anthropogenic heating on the surface temperature in the Tokyo metropolitan area: a numerical experiment. Atmos. Environ., 25B(2), pp. 155-164.

Klysik, K., 1996, Spatial and seasonal distribution of anthropogenic heat emission in Lodz, Poland. Atmos. Environ., 30, pp. 3397-3404.

Kondo, J., 1994, Meteorology of the water environment - Water and heat balance of the land surface (pp. 350). Tokyo: Asakura.

Kondo, J., 2000, Atmospheric science near the ground surface (pp. 324). Tokyo: University of Tokyo Press.

Kosugi, Y., 1996, Leaf-scale analysis of the $\mathrm{CO}_{2}$ and $\mathrm{H}_{2} \mathrm{O}$ exchange processes between trees and atmosphere, Ph.D. dissertation, Kyoto Univ., Kyoto, Japan.

Monteith, J. L., Unsworth, M. H., 1990, Principles of environmental physics. Edward Arnold.

Moriwaki, R., Kanda, M., 2004, Seasonal and diurnal fluxes of radiation, heat, water vapor, and carbon dioxide over a suburban area. Journal of Applied Meteorology, 43, pp. $1700-1710$.

Nishida, K., Nemani, R. R., Running, S. W. and Glassy, J. M., 2003, An operational remote sensing algorithm of land surface evaporation. Journal of Geophysical Research, 108 (D9), pp. 4270, doi:10.1029/2002JD002062.

Offerle, B., Jonsson, P., Eliasson, I. and Grimmond, C. S. B., 2005, Urban modification of the surface energy balance in the West African Sahel: Ouagadougou, Burkina Faso. Journal of Climate, 18, pp. 3983-3995.

Oke, T. R., 1988, The urban energy balance. Prog. Phys. Geogr., 12, pp. 471-508. 
1 Pielke, R. A., McNider R. T., Segal M. and Mahrer, Y., 1983, The use of a mesoscale numerical model for evaluations of pollutant transport and diffusion in coastal regions and over irregular terrain. Bulletin of the American Meteorological Society, 64, pp. 243249.

Piringer, M., Grimmond, C. S. B., Joffre, S. M. Mestayer, P., Middleton, D. R. et al., 2002, Investigating the surface energy balance in urban areas - recent advances and future needs. Water, Air and Soil Pollution: Focus, 2, pp. 1-16.

Sailor, D. J., Dietsch, N., 2007, The urban heat island mitigation impact screening tool (MIST). Environmental Modelling and Software, 22, pp. 1529-1541.

Sailor, D. J., Lu, L., 2004, A top-down methodology for developing diurnal and seasonal anthropogenic heating profiles for urban areas. Atmospheric Environment, 38, pp. $2737-2748$.

Schroeder, T. A., Cohen, W. B., Song, C. H., Canty, M. J. and Yang, Z. Q., 2006, Radiometric correction of multi-temporal Landsat data for characterization of early successional forest patterns in western Oregon. Remote Sensing of Environment, 103, pp. $16-26$.

Silberstein, R., Held, A., Hatton, T., Viney, N. and Sivapalan, M., 2001, Energy balance of a natural jarrah (Eucalyptus marginata) forest in Western Australia: Measurements during the spring and summer. Agricultural and Forest Meteorology, 109, pp. 79-104.

Stull, R. B., 1988, An Introduction to Boundary Layer Meteorology. Kluwer Academic Publishers, Boston, 666 pp.

Snyder, W. C., Wan, Z., Zhang, Y. and Feng Y., 1998, Classification based emissivity for land surface temperature measurement from space. International Journal of Remote Sensing. 19, pp, 2753-2774.

Tanaka, H., Ota, T., Hiyama, T. and Maximov, T. C., 2000, Seasonal variation of photosynthesis and transpiration properties of a boreal deciduous forest: Analysis using a single layer canopy model. J. Jpn. For. Soc., 82, pp. 259-267.

Toda, M., Ote, N., Tani M., Tanaka, H., Musiake, K., Aoki, M. and Boonyawat, S., 2000, Diurnal and seasonal variations of $\mathrm{CO}_{2}$ exchange processes over typical land covers in tropical monsoon region. J. Jpn. Soc. Hydrol. Water Resour., 13, pp. 276- 290.

Wang, J., Ma,Y., Menenti, M., Bastiaanssen, W.G.M. and Mitsuta, Y., 1995, The scaling-up of processes in the heterogeneous land-scape of HEIFE with the aid of satellite remote sensing. J. Met. Soc. of Japan, 73, pp. 1235-1244. 
1 Weng, Q., 2009, Thermal infrared remote sensing for urban climate and environmental studies: Methods, applications, and trends. ISPRS Journal of Photogrammetry and Remote Sensing, 64, pp. 335-344.

4 White, M. A., Thornton, P. E., Running, S. W. and Nemani, R. R., 2000, Parameterization and sensitivity analysis of the BIOME-BGC terrestrial ecosystem model: Net primary production controls, Earth Interact., 4, paper 3, 85 pp.

7 Yasuda, N., 1995, Fundamental atmospheric science (pp. 204).Tokyo: Asakura.

8 Yuan, F., Bauer M. E., 2007, Comparison of impervious surface area and normalized difference vegetation index as indicators of surface urban heat island effects in Landsat imagery. Remote Sensing of Environment, 106, pp. 375-386.

Zhang, Y. S., Inakwu O.A. Odeh, Han, C. F., 2009, Bi-temporal characterization of land surface temperature in relation to impervious surface area, NDVI and NDBI, using a sub-pixel image analysis. International Journal of Applied Earth Observation and Geoinformation, 11, pp. 256-264.

Zhang, X., Aono,Y., Monji, N., 1998, Spatial variability of urban surface heat fluxes estimated from Landsat TM data under summer and winter conditions. Journal of Agricultural Meteorology, 54, pp. 1-11. 
Table 1 Meteorological parameters used to calculate heat fluxes in Fuzhou

\begin{tabular}{ccc}
\hline Variables & June 15,1989 (GMT 3:00) & March 4, 2001 (GMT 3:00) \\
\hline Air temperature (K) & 306.85 & 291.38 \\
Wind speed (m/s) & 1 & 9 \\
Relative humidity $(\%)$ & 52 & 23 \\
Atmospheric water vapor pressure $(\mathrm{hPa})$ & 27.5 & 4.9 \\
Saturation water vapor pressure $(\mathrm{hPa})$ & 52.31 & 20.89 \\
\hline
\end{tabular}
10

\begin{tabular}{|c|c|c|c|c|c|c|c|c|c|}
\hline & \multicolumn{2}{|c|}{ Land use type } & Forest 3 & Water 1 & Agriculture $_{6}$ & Bare land 4 & Grassland 5 & Suburban7 & Urban $_{2}$ \\
\hline \multirow{8}{*}{$\begin{array}{c}\text { June } 15 \text {, } \\
1989\end{array}$} & \multirow[t]{2}{*}{ Mean H } & & 89 & 14.3 & 19.2 & 90.7 & 60.1 & 245.2 & 338.2 \\
\hline & & SD & 46.3 & 4.5 & 4.8 & 13.3 & 20 & 42.5 & 74.4 \\
\hline & \multirow[t]{2}{*}{ Mean $H_{a s}$} & & -84.9 & -220.5 & -253 & -115.6 & -136.1 & 23.3 & 50.2 \\
\hline & & SD & 48.8 & 73.7 & 66.7 & 43.4 & 73.8 & 43.3 & 23 \\
\hline & \multirow[t]{2}{*}{ Mean LE } & & 207.2 & 111.8 & 126.7 & 59.5 & 129.2 & 49.5 & 46.3 \\
\hline & & SD & 98.3 & 37.3 & 33 & 13.7 & 55.4 & 45.7 & 21.3 \\
\hline & \multirow[t]{2}{*}{ Mean G } & & 341.3 & 0 & 271.9 & 367.1 & 365.7 & 419.7 & 421.1 \\
\hline & & SD & 163.6 & 0 & 71.5 & 84.6 & 157.4 & 129.4 & 99.8 \\
\hline \multirow{8}{*}{$\begin{array}{l}\text { March } \\
4,2001\end{array}$} & \multirow[t]{2}{*}{ Mean H } & & 28.9 & 12.4 & 72 & 182 & 53.1 & 208.3 & 452.1 \\
\hline & & SD & 61.7 & 6.9 & 36.8 & 18.9 & 17.9 & 104.1 & 124.3 \\
\hline & \multirow[t]{2}{*}{ Mean $H_{a s}$} & & -298.1 & -354.3 & -127.9 & -123.3 & -201.9 & 46.2 & 94.4 \\
\hline & & SD & 149.4 & 109.6 & 131.9 & 46.4 & 83.5 & 125 & 57.6 \\
\hline & \multirow[t]{2}{*}{ Mean LE } & & 321.1 & 292.3 & 237.6 & 39.5 & 236.5 & 25.3 & 22.8 \\
\hline & & SD & 127.7 & 90.1 & 108.9 & 5.8 & 75 & 81 & 32.1 \\
\hline & \multirow[t]{2}{*}{ Mean G } & & 304.4 & 0 & 320.5 & 284.7 & 323 & 348.3 & 354.6 \\
\hline & & SD & 121.5 & 0 & 147.3 & 42.4 & 102.6 & 139.1 & 89.8 \\
\hline
\end{tabular}

11

12

13

14

15

Table 4 Mean $H$ and $H_{a s}$ of percent ISA categories and associated standard deviation (SD) in urban and suburban areas in 1989 and $2001 \quad\left(\mathrm{~W} / \mathrm{m}^{2}\right)$

\begin{tabular}{lcccccccc}
\hline $\begin{array}{l}\text { Category of } \\
\text { percent ISA }\end{array}$ & $\begin{array}{c}\text { Mean } \\
1989 H\end{array}$ & $\begin{array}{c}\text { SD of } \\
1989 \text { of } \\
H\end{array}$ & $\begin{array}{c}\text { Mean } \\
1989 H_{a s}\end{array}$ & $\begin{array}{c}\text { SD of } \\
1989 \\
\text { of } H_{a s}\end{array}$ & $\begin{array}{c}\text { Mean } \\
2001 H\end{array}$ & $\begin{array}{c}\text { SD of } \\
2001 \text { of } \\
H\end{array}$ & $\begin{array}{c}\text { Mean } \\
2001 \\
H_{a s}\end{array}$ & $\begin{array}{c}\text { SD of } \\
2001 \text { of } \\
H_{a s}\end{array}$ \\
\hline$<10 \%$ ISA & 15.7 & 0.4 & -279.2 & 7.3 & 23.2 & 0.9 & -381.0 & 1.8 \\
10-30\% ISA & 24.6 & 0.24 & -91.1 & 2.3 & 40.3 & 0.39 & -120.5 & 0.39 \\
30-50\% ISA & 45.5 & 1.7 & 16.8 & 6.0 & 64.5 & 1.9 & 32.1 & 2.9 \\
$>50 \%$ ISA & 214.6 & 76.5 & 42.2 & 35.0 & 260.2 & 137.1 & 103.5 & 88.5 \\
50-70\% ISA & 71.2 & 5.1 & 23.7 & 9.1 & 83.9 & 7.7 & 66.4 & 16.7 \\
70-90\% ISA & 99.7 & 10.2 & 43.2 & 18.0 & 114.0 & 18.9 & 99.3 & 35.7 \\
$>90 \%$ ISA & 301.2 & 76.1 & 81.0 & 28.7 & 365.1 & 136.4 & 169.4 & 78.0 \\
\hline
\end{tabular}

\begin{tabular}{cccc}
\hline Land cover & $d(\mathrm{~m})$ & $Z_{o m}(\mathrm{~m})$ & $Z_{\text {oh }}(\mathrm{m})$ \\
\hline Water & 0 & 0.001 & 0.0001 \\
Bare soil & 0 & 0.01 & 0.001 \\
Field & 0.02 & 0.004 & 0.0004 \\
Grass & 0.133 & 0.026 & 0.0026 \\
Forest & 4 & 0.78 & 0.078 \\
Urban & 7.5 & 1.5 & 0.15 \\
Suburban & 2.5 & 0.5 & 0.05 \\
\hline
\end{tabular}

Table 3 Mean and standard deviation (SD) of heat fluxes $\left(\mathrm{W} / \mathrm{m}^{2}\right)$ of different land cover types in 1989 and

Table 2 Zero plane displacement height $\mathrm{d}$ and roughness lengths $Z_{o m}$ and $Z_{\text {oh }}$ for land cover types in Fuzhou 
Table 5 Ratios of heat fluxes to the net radiation of the present study and measured previously in urban areas

\begin{tabular}{|c|c|c|c|c|c|c|}
\hline $\begin{array}{l}\text { Original } \\
\text { reference }\end{array}$ & Land use & $\begin{array}{l}\text { Height of } \\
\text { measurement }(\mathrm{m})\end{array}$ & Observation period & $\mathrm{H} / \mathrm{R}_{\mathrm{n}}$ & $\mathrm{LE} / \mathrm{R}_{\mathrm{n}}$ & $\mathrm{G} / \mathrm{R}_{\mathrm{n}}$ \\
\hline \multirow[t]{4}{*}{ Present study } & Urban (average) & - & Mar. 4, 2001(spring) & 0.53 & 0.04 & 0.57 \\
\hline & Urban (average) & - & Jun. 15,1989 (summer) & 0.49 & 0.06 & 0.56 \\
\hline & Suburban (average) & - & Mar. 4, 2001(spring) & 0.37 & 0.04 & 0.57 \\
\hline & Suburban (average) & - & Jun. 15,1989 (summer) & 0.36 & 0.07 & 0.58 \\
\hline $\begin{array}{l}\text { Grimmond et } \\
\text { al., } 2004\end{array}$ & $\begin{array}{l}\text { Marseille, France(urban: } \\
86 \% \text { impervious) }\end{array}$ & 34.6 and 28.5 & $\begin{array}{l}\text { Jun. 16-Jul. 14, } 2001 \\
\text { (summer) }\end{array}$ & 0.69 & 0.17 & 0.27 \\
\hline $\begin{array}{l}\text { Christen et al., } \\
2004\end{array}$ & $\begin{array}{l}\text { Basel, Switzerland } \\
\text { (urban: } 84 \% \text { impervious) }\end{array}$ & - & $\begin{array}{l}\text { Jun. 10-Jul. 10, } 2002 \\
\text { (summer) }\end{array}$ & 0.48 & 0.18 & 0.38 \\
\hline $\begin{array}{l}\text { Moriwaki et al., } \\
2006\end{array}$ & $\begin{array}{l}\text { Tokyo, Japan (urban: } \\
71 \% \text { impervious) }\end{array}$ & - & Jul. 2001 (summer) & 0.49 & 0.28 & 0.26 \\
\hline Kato et al., 2007 & Nagoya, Japan (urban) & - & Jul. 10, 2000(summer) & 0.2 & 0.00 & 0.80 \\
\hline Kato et al, 2005 & Nagoya, Japan(suburban) & - & Jul. 10, 2000(summer) & 0.56 & 0.1 & 0.39 \\
\hline $\begin{array}{l}\text { Offerle et al., } \\
2005\end{array}$ & $\begin{array}{l}\text { Ouagadougou, Burkina } \\
\text { Faso (urban: } 40 \% \\
\text { impervious ) }\end{array}$ & - & $\begin{array}{l}\text { Feb. 8-20, } 2003 \\
\text { (spring) }\end{array}$ & 0.41 & 0.11 & 0.47 \\
\hline
\end{tabular}

3 
(a) June 15, 1989

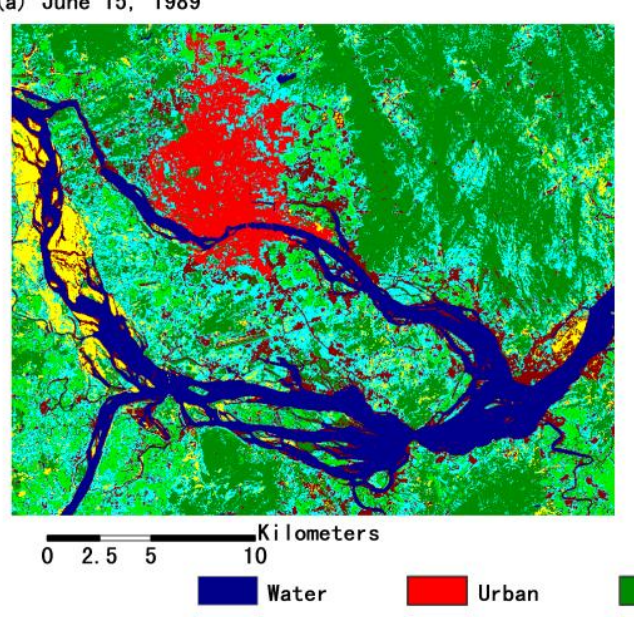

(b) March 4, 2001

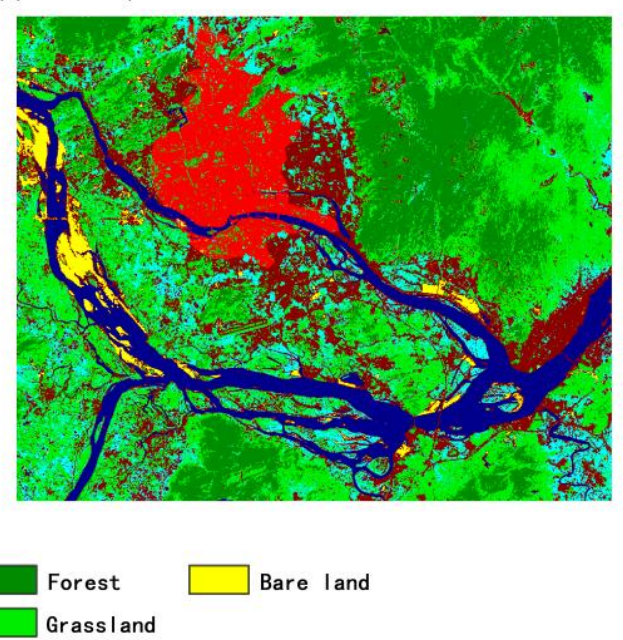

Fig. 2 Land cover types of the study area derived from the TM image acquired in 1989 (left) and ETM+ image acquired in 2001 (right). 
2

3

4

5

6

7

8

9

10

11

12

13

14

15

16

17

18

19

20

21

22

23

24

25

26

27

28

29

30

31

32

33

34

35

36

37

38

39

40

41

42

43

44

45

46

47

48

49 (a)

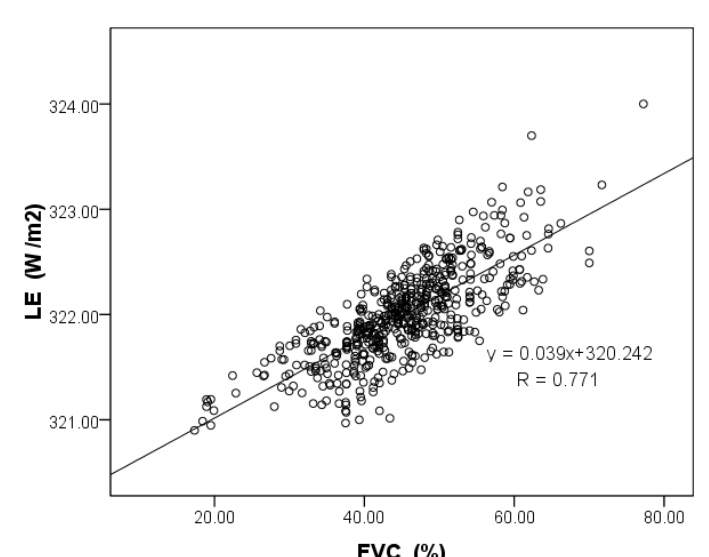

(c)

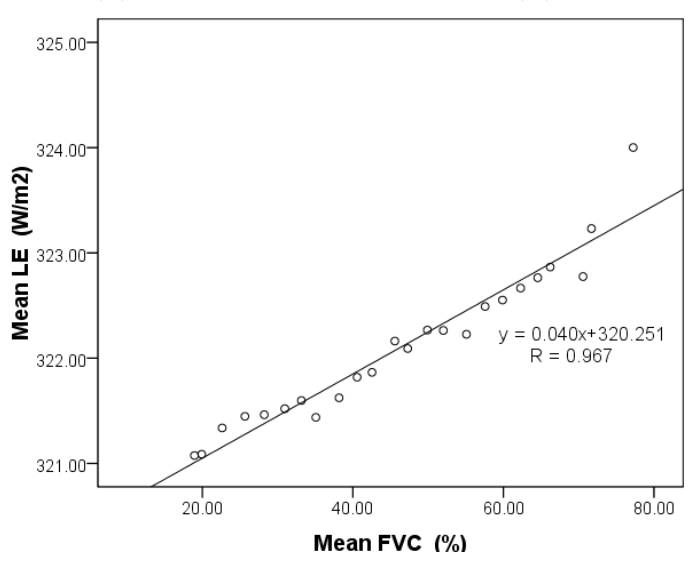

(b)
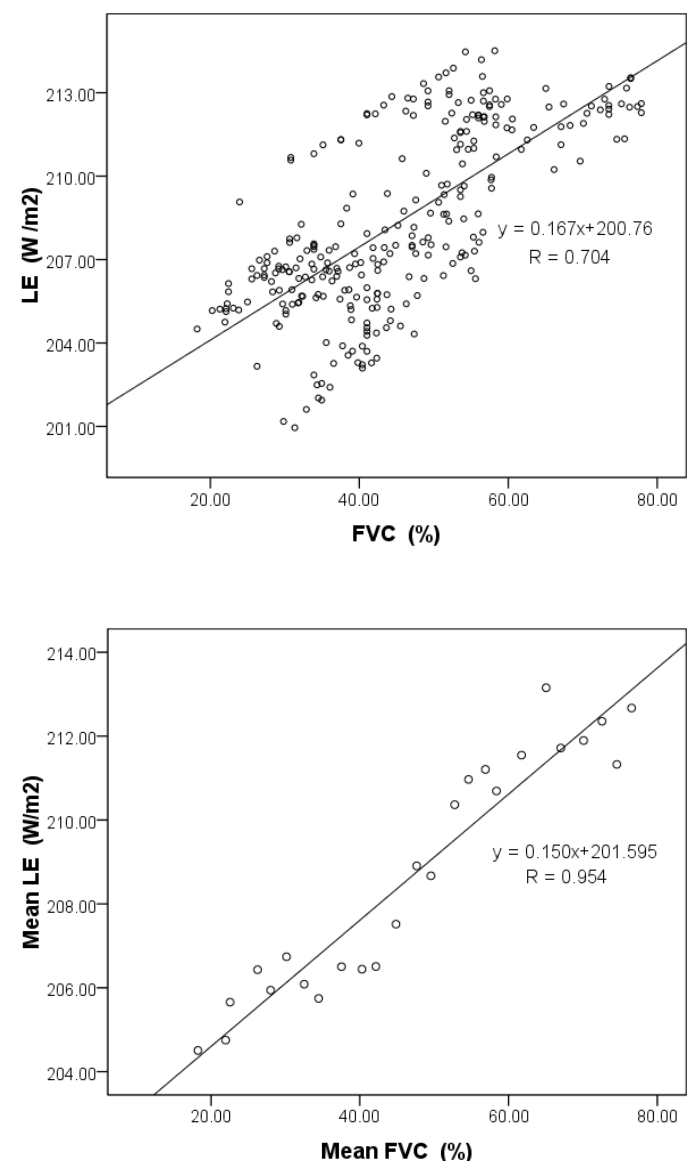

Fig. 3 Scatterplots of $L E$ versus FVC (a and b) and linear plots of mean $L E$ versus FVC (c and d) in urban area 
(b) $L E$ on March 4, 2001
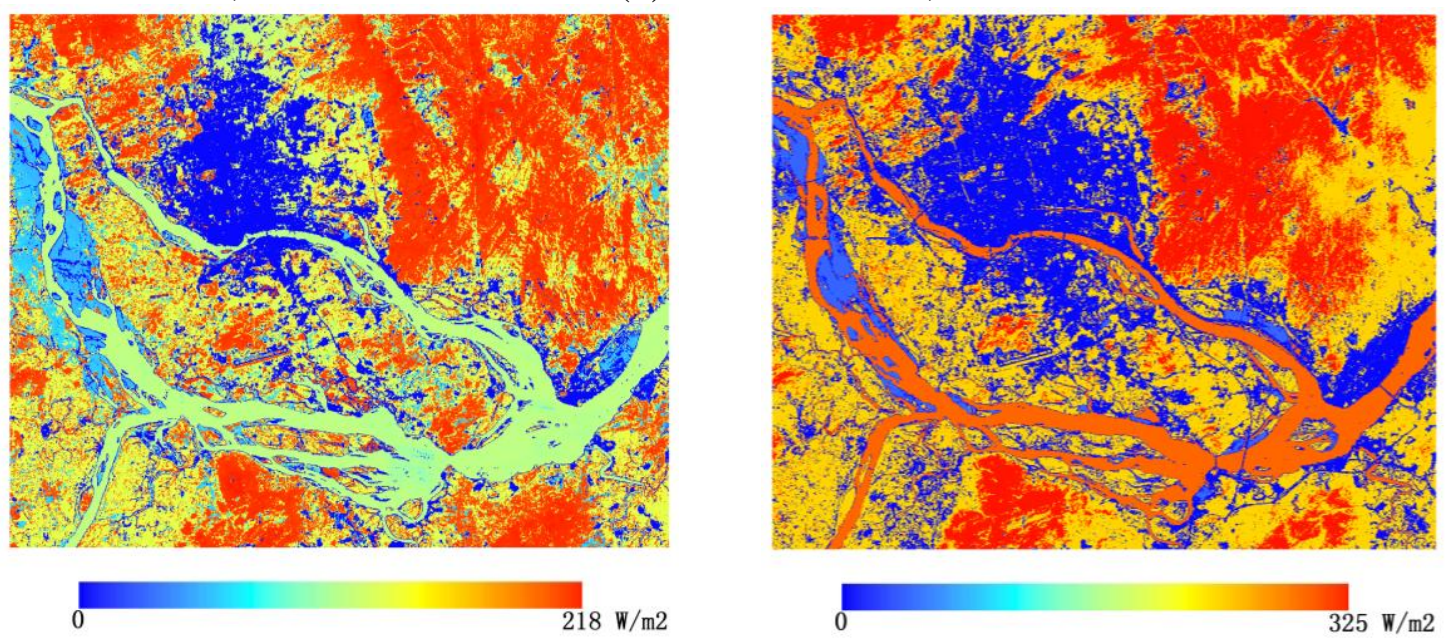

(c) $L E$ corrected by FVC on June 15, 1989

(d) $L E$ corrected by FVC on March 4, 2001
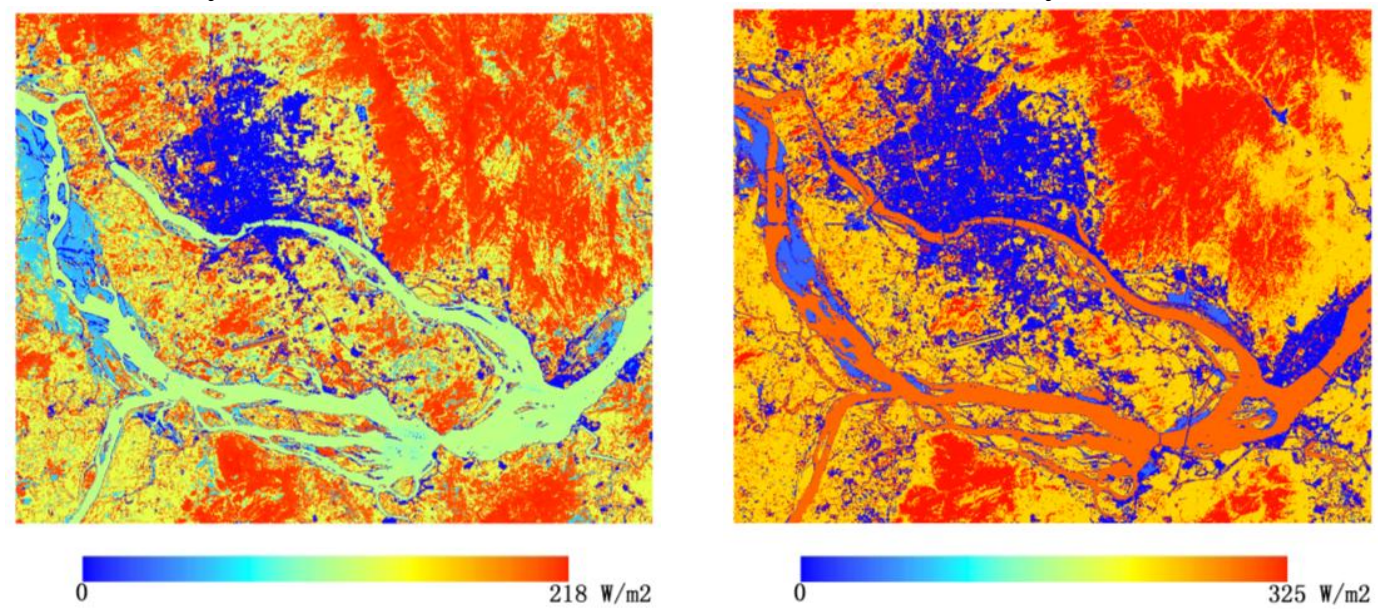

4

6

7

8

9

10

11

12

13

14

15

16

17

18

19

20

Fig. 4: Maps of $L E$ retrieved from Landsat images using two different methods and two acquisition dates. (a) $L E$ for impervious surfaces set to zero using a crisp classification method; image acquired on June 15, 1989; (b) $L E$ for impervious surfaces set to zero using a crisp classification method; image acquired on March 4, 2001; (c) $L E$ for impervious surfaces corrected using FVC from spectral unmixing; image acquired on June 15, 1989; (d) $L E$ for impervious surfaces corrected using FVC from spectral unmixing; image acquired on March 4, 2001. 

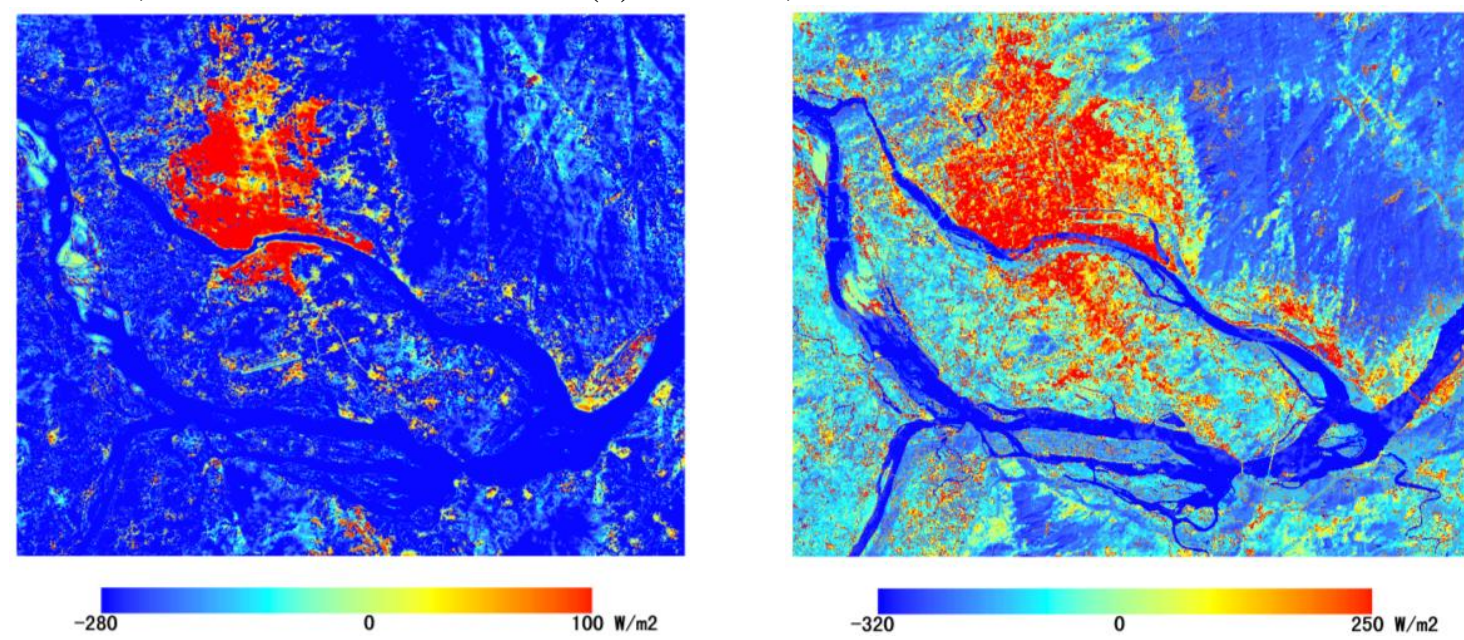

Fig. 5 Anthropogenic heat discharge from sensible heat flux on (a) June 15, 1989 and (b) March 4, 2001

7

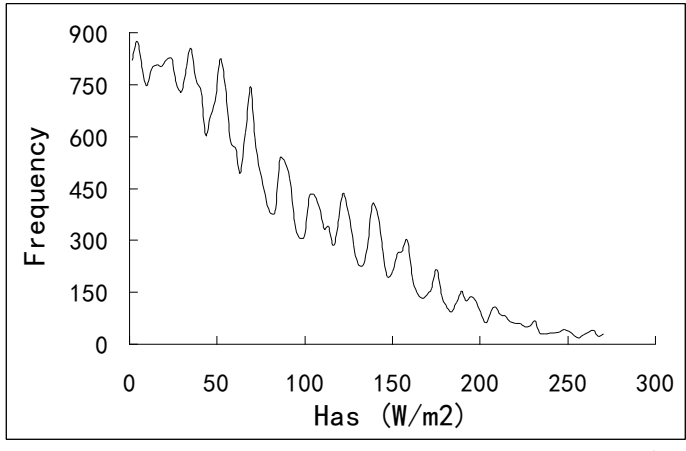

(a) June 15,1989

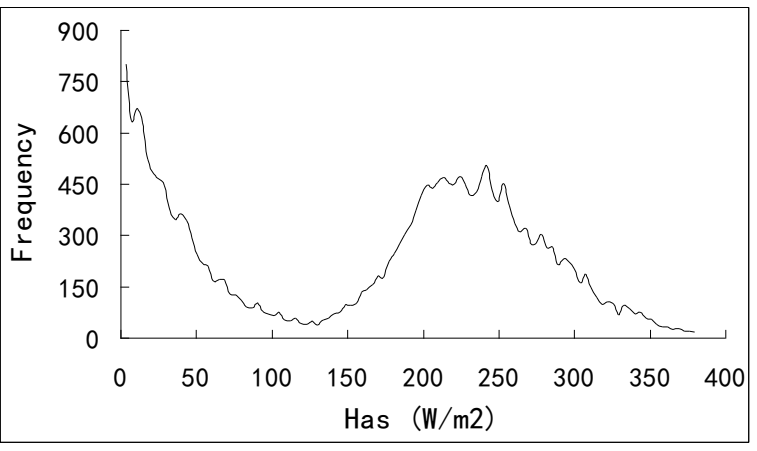

(b) March 4, 200

10 Fig. 6 Frequency distribution of anthropogenic heat flux of the medium- and high- density

11 urban areas

13

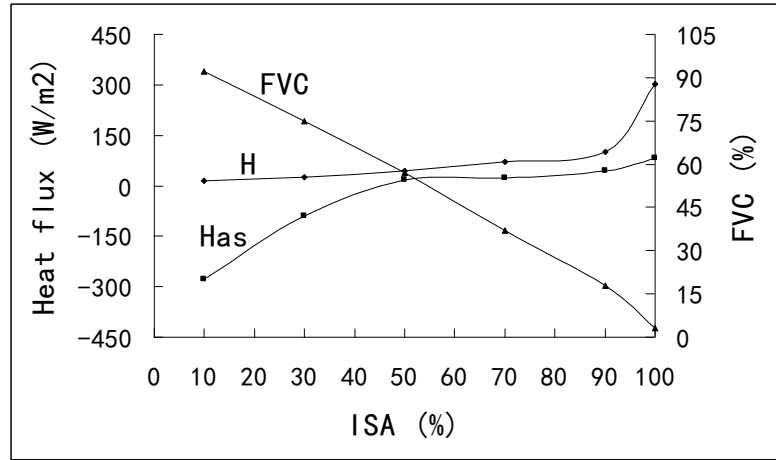

(a) June 15,1989

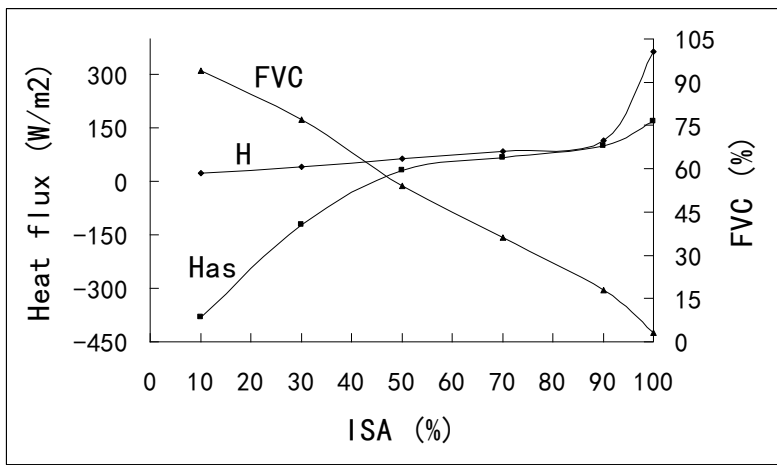

(b) March 4, 2001

16 Fig. 7 The 1989 and 2001 mean $H, H_{a s}$ and FVC for each percent ISA category in urban and 17 sub-urban areas. 